\title{
On the shear stress function and the critical value of the Blasius problem
}

\author{
GC Yang*, YZ Xu and LF Dang
}

${ }^{*}$ Correspondence: gcyang@cuit.edu.cn

College of Mathematics, Chengdu University of Information

Technology, Chengdu, 610225, P.R. China

\begin{abstract}
The Blasius problem has been used to describe the steady two-dimensional flow of a slightly viscous incompressible fluid past a flat plate moving at a constant speed $\beta$; and it is well known that there exists the critical value $\beta^{*}<0$ such that it has at least one solution for each $\beta \geq \beta^{*}$ and has no positive solution for $\beta<\beta^{*}$. The known numerical result shows $\beta^{*} \doteq-0.3541$. In this paper, by the study of the integral equation equivalent to the Blasius problem, we obtain the relation between the velocity function $f^{\prime}$ and the shear stress functions $f^{\prime \prime}$, upper and lower bounds of $\left\|f^{\prime \prime}\right\|$ and a new lower bound of $\beta^{*}$. In particular, $\sqrt[4]{27} / 9 \leq\left\|f^{\prime \prime}\right\| \leq \sqrt{3} / 3, \beta^{*}>-0.45$. Regarding $\beta^{*}$, previous results presented a lower bound -0.5 and an upper bound -0.18733 .
\end{abstract}

Keywords: Blasius problem; shear stress function; critical value; upper and lower bounds; Crocco equation

\section{Introduction}

The Blasius problem [1] arising in the boundary layer problems in fluid mechanics

$$
f^{\prime \prime \prime}(\eta)+f(\eta) f^{\prime \prime}(\eta)=0 \quad \text { on }[0, \infty)
$$

subject to the boundary conditions

$$
f(0)=0, \quad f^{\prime}(0)=\beta \quad \text { and } \quad f^{\prime}(\infty)=1,
$$

has been used to describe the steady two-dimensional flow of a slightly viscous incompressible fluid past a flat plate. It also arises in the study of the mixed convection in porous media [2], where $\eta$ is the similarity boundary layer ordinate, $f(\eta)$ is the similarity stream function, $f^{\prime}(\eta)$ and $f^{\prime \prime}(\eta)$ are the velocity and the shear stress functions, respectively. The case of $\beta<0$ corresponds to a flat plate moving at a steady speed opposite to that of a uniform mainstream [3].

Regarding the analytic study of the Blasius problem (1.1)-(1.2), Weyl [4] proved that (1.1)(1.2) has one and only one solution for $\beta=0$; Coppel [5] studied the case of $\beta>0$; the cases of $0<\beta<1$ [6] and $\beta>1$ [7] were also investigated, respectively. Also, see [8]. In 1986, Hussaini and Lakin [9] indicated that there exists a critical value $\beta^{*}<0$ such that (1.1)-(1.2) has at least a solution for $\beta \geq \beta^{*}$ and no solution for $\beta<\beta^{*}$. A lower bound was presented with $\beta^{*} \geq-1 / 2=-0.5$ and numerical results showed $\beta^{*} \doteq-0.3541$ [9]. In

\section{Springer}

(c) 2012 Yang et al.; licensee Springer. This is an Open Access article distributed under the terms of the Creative Commons Attribution License (http://creativecommons.org/licenses/by/2.0), which permits unrestricted use, distribution, and reproduction in any medium, provided the original work is properly cited. 
2008, Brighi, Fruchard and Sari [10] summarized historical study on the Blasius problem and analyzed the case $\beta<0$ in detail, in which the shape and the number of solutions were determined. In 2010, Yang [11] obtained an upper bound $\beta^{*}<-18733 / 10^{6}=-0.18733$. The Blasius problem is a special case of the Falkner-Skan equation, for $\beta=0$, we may refer to [12-15] for some recent results on the Falkner-Skan equation.

An open question is: What exactly is $\beta^{*}$ ? And what properties does the shear stress function $f^{\prime \prime}(\eta)$ have? To our knowledge, there is little study on it. By the study of the integral equation that is equivalent to the Blasius problem, in this paper, we present the relation between $f^{\prime}$ and $f^{\prime \prime}$, upper and lower bounds of $\left\|f^{\prime \prime}\right\|$ and new lower bounds of $\beta^{\prime \prime}$. In particular, $\sqrt[4]{27} / 9 \leq\left\|f^{\prime \prime}\right\| \leq \sqrt{3} / 3, \beta^{*}>-0.45$.

\section{Upper and lower bounds of $f^{\prime \prime}$}

Noticing the basic fact in [10] that if $f$ is a solution of (1.1)-(1.2), then $f^{\prime \prime}>0$ for $\eta \in[0, \infty)$, we use the so-called Crocco transformation $[9,10]$, which consists of choosing $t=f^{\prime}$ as an independent variable and expressing $z=f^{\prime \prime}$ as a function of $t$, to change (1.1)-(1.2) to the Crocco equation [10]

$$
\frac{d^{2} z}{d t^{2}}=-\frac{t}{z}, \quad \beta \leq t<1
$$

with the boundary conditions

$$
z^{\prime}(\beta)=0, \quad z(1)=0 .
$$

Integrating (2.1) from $\beta$ to $t$, we have by (2.2)

$$
z^{\prime}(t)=-\int_{\beta}^{t} \frac{s}{z(s)} d s \quad \text { on }[\beta, 1)
$$

Integrating (2.3) from $t$ to 1 , we obtain the following integral equation that is equivalent to $(1.1)-(1.2)[10,11]$ :

$$
z(t)=\int_{t}^{1} \frac{s(1-s)}{z(s)} d s+(1-t) \int_{\beta}^{t} \frac{s}{z(s)} d s:=A z(t)+(1-t) B z(t),
$$

where $z(t) \in C[\beta, 1]$ and $z(t)>0$ for $t \in[\beta, 1)$.

Since $\beta^{*} \geq-\frac{1}{2}$, our work is restricted to the case of $-\frac{1}{2} \leq \beta<0$ and begins with the following lemma.

Theorem 2.1 Let $z$ be a solution of (2.4), then

(i) $\|z\| \leq \sqrt{h(\beta)}$;

(ii) $-\frac{\beta^{3}}{3\|z\|} \leq z(0) \leq \frac{3 h(\beta)+\beta^{3}}{3 \sqrt{h(\beta)}}$, where $\|z\|=\max \{z(t): t \in[\beta, 1]\}, h(\beta)=\frac{1}{3}\left(1-3 \beta^{2}-2 \beta^{3}\right)$.

Proof Let $\tilde{t} \in[\beta, 1]$ such that $\|z\|=z(\tilde{t})=\max \{z(t): t \in[\beta, 1]\}$. By (2.3), we know $z^{\prime}(t)>0$ for $t \in(\beta, 0]$, and then $z(t)$ is strictly increasing $(\beta, 0]$. This, together with $z(\tilde{t})>0$, implies $0<\tilde{t}<1$. From (2.1), we know $z^{\prime \prime}(t) z(t)=-t$. Integrating this equality from $\beta$ to $\tilde{t}$, we have

$$
\int_{\beta}^{\tilde{t}} z^{\prime \prime}(s) z(s) d s=-\int_{\beta}^{\tilde{t}} s d s=-\frac{\tilde{t}^{2}-\beta^{2}}{2} .
$$


Noticing that $z^{\prime}(\tilde{t})=z^{\prime}(\beta)=0$, we obtain

$$
\int_{\beta}^{\tilde{t}} z^{\prime \prime}(s) z(s) d s=\left.z^{\prime}(s) z(s)\right|_{\beta} ^{\tilde{t}}-\int_{\beta}^{\tilde{t}} z^{\prime 2}(s) d s=-\int_{\beta}^{\tilde{t}} z^{\prime 2}(s) d s \leq 0 .
$$

Consequently, $|\beta| \leq \tilde{t}$.

(i) From $z^{\prime}(\tilde{t})=-\int_{\beta}^{\tilde{t}} \frac{s}{z(s)} d s=-B z(\tilde{t})$, we know $B z(t) \geq B z(\tilde{t})=0$ for $t \in[\tilde{t}, 1)$. This implies $A z(t) \leq z(t), t \in[\tilde{t}, 1)$.

By $A^{\prime} z(t)=-\frac{t(1-t)}{z(t)}$, we have

$$
A z(t)(-A z(t))^{\prime} \leq z(t)(-A z(t))^{\prime} \leq t(1-t) \quad \text { for } t \in[\tilde{t}, 1)
$$

Noticing that $z(\tilde{t})=A z(\tilde{t})$ and $z(1)=0$, integrating (2.5) from $\tilde{t}$ to 1 , we have by $|\beta| \leq \tilde{t}$

$$
\begin{aligned}
\|z\|^{2} & =[A z(\tilde{t})]^{2} \leq 2 \int_{\tilde{t}}^{1} s(1-s) d s \leq 2 \int_{|\beta|}^{1} s(1-s) d s \\
& =\frac{1}{3}\left(1-3 \beta^{2}-2 \beta^{3}\right)=h(\beta) .
\end{aligned}
$$

(ii) Integrating (2.3) from $\beta$ to 0 , we have

$$
z(0)=-\int_{\beta}^{0} B z(t) d t+z(\beta) \geq \int_{\beta}^{0} \int_{\beta}^{t} \frac{-s}{\|z\|} d s d t+z(\beta) \geq-\frac{\beta^{3}}{3\|z\|},
$$

which implies that the left inequality of (ii) holds.

Noticing that $|\beta| \leq \tilde{t}$ and utilizing (2.1) and (2.2), we know

$$
z(\tilde{t})-z(0)=\int_{0}^{\tilde{t}} \int_{t}^{\tilde{t}} \frac{s}{z(s)} d s d t \geq \int_{0}^{|\beta|} \int_{t}^{|\beta|} \frac{s}{\|z\|} d s d t=-\frac{\beta^{3}}{3\|z\|} .
$$

And then $z(0) \leq\|z\|+\frac{\beta^{3}}{3\|z\|} \leq \sqrt{h(\beta)}+\frac{\beta^{3}}{3 \sqrt{h(\beta)}}=\frac{3 h(\beta)+\beta^{3}}{3 \sqrt{h(\beta)}}$. Hence, (ii) holds.

Let

$$
l(t)= \begin{cases}\frac{1}{6 \sqrt{h(\beta)}}\left(t+t^{2}-2 \beta^{3}\right)(1-t), & t \in[0,1], \\ \frac{1}{6 \sqrt{h(\beta)}}\left(3 \beta^{2} t-t^{3}-2 \beta^{3}\right), & t \in[\beta, 0)\end{cases}
$$

and

$$
u(t)= \begin{cases}3 \sqrt{h(\beta)}\left(2 \ln \frac{2}{1+t}+(1-t) \ln \frac{1+t}{1-t}\right), & t \in[0,1], \\ \frac{6 h(\beta)+2 \beta^{3}+3 \beta^{2} t-t^{3}}{3 \sqrt{h(\beta)}}, & t \in[\beta, 0) .\end{cases}
$$

Utilizing Theorem 2.1, we can obtain upper and lower bounds of $z$ as follows.

Theorem 2.2 Let $z$ be a solution of (2.4), then $l(t) \leq z(t) \leq u(t)$ for $t \in[\beta, 1]$. 
Proof For $t \in[\beta, 0)$, we have by (2.3) and Theorem 2.1(i)

$$
z^{\prime}(t)=-B z(t) \geq \int_{\beta}^{t} \frac{-s}{\sqrt{h(\beta)}} d s=\frac{\beta^{2}-t^{2}}{2 \sqrt{h(\beta)}} \quad \text { on }[\beta, 0) .
$$

From this, we have

$$
z(t) \geq \int_{\beta}^{t} \frac{\beta^{2}-s^{2}}{2 \sqrt{h(\beta)}} d s+z(\beta) \geq \frac{1}{6 \sqrt{h(\beta)}}\left(3 \beta^{2} t-t^{3}-2 \beta^{3}\right) .
$$

Hence, $z(t) \geq l(t)$ for $t \in[\beta, 0)$.

For $t \in[0,1]$, let $\varepsilon>0$, we define a function $h_{\varepsilon}(t)$ as follows:

$$
h_{\varepsilon}(t)=\frac{1}{\|z\|+\varepsilon}\left(-\frac{\beta^{3}}{3}(1-t)+\int_{0}^{1} G(t, s) s d s\right) \quad \text { for } t \in[0,1] \text {, }
$$

where $G(t, s)$ is the Green function for $w^{\prime \prime}(t)=0$ with boundary conditions $w(0)=0=w(1)$ defined by

$$
G(t, s)= \begin{cases}t(1-s), & 0 \leq t \leq s \leq 1 \\ s(1-t), & 0 \leq s \leq t \leq 1\end{cases}
$$

Next, we prove $z(t) \geq h_{\varepsilon}(t)$ for $t \in[0,1]$.

In fact, if there exists $t_{0} \in[0,1]$ such that $z\left(t_{0}\right)<h_{\varepsilon}\left(t_{0}\right)$, since $h_{\varepsilon}(1)=0=z(1), h_{\varepsilon}(0)=$ $-\frac{\beta^{3}}{3(\|z\|+\varepsilon)}<-\frac{\beta^{3}}{3\|z\|} \leq z(0)$ by Theorem 2.1(ii), then $t_{0} \in(0,1)$ and there exists an interval $(a, b) \subseteq(0,1)$ such that

$$
t_{0} \in(a, b), z(t)<h_{\varepsilon}(t) \quad \text { for } t \in(a, b), z(a)=h_{\varepsilon}(a), z(b)=h_{\varepsilon}(b) .
$$

Let $\varphi(t)=h_{\varepsilon}(t)-z(t)$, then $\varphi(a)=0=\varphi(b)$ and $\varphi\left(t_{0}\right)>0$. Let $\xi \in(a, b)$ such that $\varphi(\xi)=$ $\max \{\varphi(t): t \in[a, b]\}$, then $\varphi^{\prime \prime}(\xi) \leq 0$. On the other hand, we know easily that

$$
\varphi^{\prime \prime}(t)=h_{\varepsilon}^{\prime \prime}(t)-z^{\prime \prime}(t)=-\frac{t}{\|z\|+\varepsilon}+\frac{t}{z(t)}>0 \quad \text { for } t \in(a, b) .
$$

Then $\varphi^{\prime \prime}(\xi)>0$, a contradiction.

Taking $\varepsilon \rightarrow 0$, we have

$$
z(t) \geq \frac{1}{\|z\|}\left(-\frac{\beta^{3}}{3}(1-t)+\int_{0}^{1} G(t, s) s d s\right) \text { for } t \in[0,1] .
$$

Since $\int_{0}^{1} G(t, s) s d s=\frac{\left(t+t^{2}\right)(1-t)}{6}$, we have

$$
z(t) \geq \frac{1}{6\|z\|}\left(t+t^{2}-2 \beta^{3}\right)(1-t)
$$

Theorem 2.1(i) leads to

$$
z(t) \geq \frac{1}{6 \sqrt{h(\beta)}}\left(t+t^{2}-2 \beta^{3}\right)(1-t)=l(t) \quad \text { for } t \in[0,1]
$$


Finally, we prove $z(t) \leq u(t)$. For $t \in[\beta, 0)$, integrating (2.6) from $t$ to 0 , we obtain $z(0)-$ $z(t) \geq \frac{t^{3}-3 \beta^{2} t}{6 \sqrt{h(\beta)}}$. Then by Theorem 2.1(ii),

$$
z(t) \leq z(0)-\frac{t^{3}-3 \beta^{2} t}{6 \sqrt{h(\beta)}} \leq \frac{3 h(\beta)+\beta^{3}}{3 \sqrt{h(\beta)}}-\frac{t^{3}-3 \beta^{2} t}{6 \sqrt{h(\beta)}}=u(t) .
$$

For $t \in[0,1)$, since $z(t) \geq l(t) \geq \frac{1}{6 \sqrt{h(\beta)}}\left(t+t^{2}\right)(1-t):=l_{0}(t)$, we have

$$
\begin{aligned}
z(t) & \leq \int_{t}^{1} \frac{s(1-s)}{z(s)} d s+(1-t) \int_{0}^{t} \frac{s}{z(s)} d s \\
& \leq \int_{t}^{1} \frac{s(1-s)}{l_{0}(s)} d s+(1-t) \int_{0}^{t} \frac{s}{l_{0}(s)} d s \\
& =6 \sqrt{h(\beta)}\left(\int_{t}^{1} \frac{1}{1+s} d s+(1-t) \int_{0}^{t} \frac{1}{1-s^{2}} d s\right) \\
& =3 \sqrt{h(\beta)}\left(2 \ln \frac{2}{1+t}+(1-t) \ln \frac{1+t}{1-t}\right)=u(t) .
\end{aligned}
$$

Combining Theorems 2.1 and 2.2, we obtain

Corollary 2.1 Let $z$ be a solution of $(2.4)$, then $\sqrt[4]{h(\beta)} \sqrt{l\left(\frac{\sqrt{3+6 \beta^{3}}}{3}\right)} \leq\|z\| \leq \sqrt{h(\beta)}$. In particular, $\frac{\sqrt[4]{27}}{9} \leq\|z\| \leq \frac{\sqrt{3}}{3}$.

Proof Let $g_{\beta}(t)=\left(t+t^{2}-2 \beta^{3}\right)(1-t)$, by (2.8), we have $z(t) \geq \frac{1}{6\|z\|} g_{\beta}(t)$ on $[0,1]$. From $g_{\beta}^{\prime}(\hat{t})=0$, we obtain $\hat{t}=\frac{\sqrt{3+6 \beta^{3}}}{3}$, and then $\max \left\{g_{\beta}(t): t \in[0,1]\right\}=g_{\beta}\left(\frac{\sqrt{3+6 \beta^{3}}}{3}\right)$. Hence, $\|z\| \geq \frac{1}{6\|z\|} g_{\beta}\left(\frac{\sqrt{3+6 \beta^{3}}}{3}\right)$. This, together with $g_{\beta}\left(\frac{\sqrt{3+6 \beta^{3}}}{3}\right)=6 \sqrt{h(\beta)} l\left(\frac{\sqrt{3+6 \beta^{3}}}{3}\right)$, implies $\|z\| \geq$ $\sqrt[4]{h(\beta)} \sqrt{l\left(\frac{\sqrt{3+6 \beta^{3}}}{3}\right)}$. The right hand is from Theorem 2.1(i).

Since $h(\sigma) \leq h(0)=\frac{1}{3}$ for $\sigma \geq 0$, hence $\|z\| \leq \sqrt{h(0)}=\frac{\sqrt{3}}{3}$.

Since $g_{\beta}(t) \geq t(1+t)(1-t):=g_{0}(t)$ for $t \in[0,1]$, by $g_{0}^{\prime}(\hat{t})=0$, we have $\hat{t}=\frac{\sqrt{3}}{3}$. Hence, $\max \left\{g_{0}(t): t \in[0,1]\right\}=g_{0}(\hat{t})=\frac{2 \sqrt{3}}{9}$. By $(2.8)$, we obtain

$$
\|z\| \geq z(t) \geq \frac{g_{0}(t)}{6\|z\|} \quad \text { on }[0,1]
$$

From this, we have $\|z\| \geq \frac{g_{0}(\hat{t})}{6\|z\|}=\frac{\sqrt{3}}{27\|z\|}$, i.e., $\|z\| \geq \frac{\sqrt[4]{27}}{9}$.

Based on Theorem 2.2, Corollary 2.1, $f^{\prime}(\eta)=t$ and $f^{\prime \prime}(\eta)=z(t)$, we obtain the relation between the velocity function $f^{\prime}$ and the shear stress functions $f^{\prime \prime}$, upper and lower bounds of $\left\|f^{\prime \prime}\right\|=\sup \left\{f^{\prime \prime}(\eta): \eta \in[0, \infty\}\right.$.

Theorem 2.3 Let $f$ be a solution of (1.1)-(1.2), then

(i) $l\left(f^{\prime}\right) \leq f^{\prime \prime} \leq u\left(f^{\prime}\right)$ for $\eta \in[0, \infty)$;

(ii) $\sqrt[4]{h(\beta)} \sqrt{l\left(\frac{\sqrt{3+6 \beta^{3}}}{3}\right)} \leq\left\|f^{\prime \prime}\right\| \leq \sqrt{h(\beta)}$. Specially, $\frac{\sqrt[4]{27}}{9} \leq\left\|f^{\prime \prime}\right\| \leq \frac{\sqrt{3}}{3}$. 
Remark 2.1 There exists very little study on the upper and lower bounds of $f^{\prime \prime}(\eta)$; Theorem 2.3 fills this gap. Other studies can be found in $[9,10]$.

\section{New lower bound of $\boldsymbol{\beta}^{*}$}

To obtain a better lower bound of $\beta^{\prime \prime}$, we first prove

Theorem 3.1 Let $z$ be a solution of (2.4), then

$$
\int_{\beta}^{1}(1-s) z^{\prime 2}(s) d s \geq \frac{1}{1440 h(\beta)}\left(60 \beta^{6}-192 \beta^{5}+7\right) .
$$

Proof Firstly, we prove

$$
\left|z^{\prime}(t)\right| \geq \frac{\left|t^{2}-\tilde{t}^{2}\right|}{2 \sqrt{h(\beta)}} \quad \text { on }[0,1]
$$

Integrating (2.1) from $t$ to $\tilde{t}$, we obtain by $z^{\prime}(\tilde{t})=0$

$$
z^{\prime}(t)=\int_{t}^{\tilde{t}} \frac{s}{z(s)} d s \geq \int_{t}^{\tilde{t}} \frac{s}{\sqrt{h(\beta)}} d s=\frac{\tilde{t}^{2}-t^{2}}{2 \sqrt{h(\beta)}} \quad \text { on }[0, \tilde{t}) .
$$

Integrating (2.1) from $\tilde{t}$ to $t$, we have by $z^{\prime}(\tilde{t})=0$

$$
z^{\prime}(t)=\int_{\tilde{t}}^{t} \frac{-s}{z(s)} d s \leq \int_{\tilde{t}}^{t} \frac{-s}{\sqrt{h(\beta)}} d s=\frac{\tilde{t}^{2}-t^{2}}{2 \sqrt{h(\beta)}} \leq 0 \quad \text { on }[\tilde{t}, 1] .
$$

Hence, (3.1) holds.

From (2.6) and (3.1), we know

$$
\begin{aligned}
\int_{\beta}^{1}(1-s) z^{\prime 2}(s) d s & =\int_{\beta}^{0}(1-s) z^{\prime 2}(s) d s+\int_{0}^{1}(1-s) z^{\prime 2}(s) d s \\
& \geq \frac{1}{4 h(\beta)}\left[\int_{\beta}^{0}(1-s)\left(\beta^{2}-s^{2}\right)^{2} d s+\int_{0}^{1}(1-s)\left(s^{2}-\tilde{t}^{2}\right)^{2} d s\right] .
\end{aligned}
$$

By

$$
\begin{aligned}
& \int_{\beta}^{0}(1-s)\left(\beta^{2}-s^{2}\right)^{2} d s=\frac{1}{30}\left(5 \beta^{6}-16 \beta^{5}\right), \\
& \int_{0}^{1}(1-s)\left(s^{2}-\tilde{t}^{2}\right)^{2} d s=\frac{1}{30}\left(1-5 \tilde{t}^{2}+15 \tilde{t}^{4}\right)
\end{aligned}
$$

and

$$
1-5 \tilde{t}^{2}+15 \tilde{t}^{4}=15\left(\tilde{t}^{2}-\frac{1}{6}\right)^{2}+\frac{7}{12} \geq \frac{7}{12}
$$


we obtain

$$
\begin{aligned}
\int_{\beta}^{1}(1-s) z^{\prime 2}(s) d s & \geq \frac{1}{120 h(\beta)}\left[\left(5 \beta^{6}-16 \beta^{5}\right)+\frac{7}{12}\right] \\
& =\frac{1}{1440 h(\beta)}\left(60 \beta^{6}-192 \beta^{5}+7\right) .
\end{aligned}
$$

Let

$$
H(\beta)=73-480 \beta^{2}+720 \beta^{4}+192 \beta^{5}-380 \beta^{6}, \quad \beta \in\left[-\frac{1}{2}, 0\right] .
$$

Since

$$
\begin{aligned}
& H^{\prime}(\beta)=-960 \beta+2880 \beta^{3}+960 \beta^{4}-2280 \beta^{5}>-960 \beta\left(1-3 \beta^{2}\right)>0 \quad \text { on }\left[-\frac{1}{2}, 0\right), \\
& H\left(-\frac{9}{20}\right)=-\frac{4396387}{3200000}<0, \quad H\left(-\frac{11}{25}\right)=\frac{55395529}{48828125}>0,
\end{aligned}
$$

then there exists a unique $\tilde{\beta} \in\left(-\frac{9}{20},-\frac{11}{25}\right)=(-0.45,-0.44)$ such that $H(\tilde{\beta})=0, H(\beta)>0$ for $\beta \in(\tilde{\beta}, 0)$ and $H(\beta)<0$ for $\beta \in\left(-\frac{1}{2}, \tilde{\beta}\right)$.

Theorem 3.2 If $\beta \leq \tilde{\beta}$, the Blasius problem (1.1)-(1.2) has no solution and then $\beta^{*}>-0.45$.

Proof The proof is by contradiction. If for some $\beta \leq \tilde{\beta},(1.1)-(1.2)$ has a solution $f$ and then (2.1) has a solution $z$. Rewrite (2.1) as follows:

$$
\left(z(t) z^{\prime}(t)\right)^{\prime}+t=z^{2}(t)
$$

Integrating this equality from $\beta$ to $t$ and noticing that $z^{\prime}(\beta)=0$, we obtain

$$
z(t) z^{\prime}(t)+\int_{\beta}^{t} s d s=\int_{\beta}^{t} z^{\prime 2}(s) d s
$$

Integrating the last equality from $\beta$ to 1 and using $z(1)=0$, we have

$$
-\frac{1}{2} z^{2}(\beta)+\int_{\beta}^{1} \int_{\beta}^{t} s d s d t=\int_{\beta}^{1} \int_{\beta}^{t} z^{\prime 2}(s) d s d t=\int_{\beta}^{1}(1-s) z^{\prime 2}(s) d s .
$$

This, together with $z(\beta)>0$ and Theorem 3.1, implies

$$
\frac{1-3 \beta^{2}+2 \beta^{3}}{6}=\int_{\beta}^{1} \int_{\beta}^{t} s d s d t>\frac{1}{1440 h(\beta)}\left(60 \beta^{6}-192 \beta^{5}+7\right) .
$$

Since

$$
\frac{1-3 \beta^{2}+2 \beta^{3}}{6}-\frac{1}{1440 h(\beta)}\left(60 \beta^{6}-192 \beta^{5}+7\right)=\frac{H(\beta)}{1440 h(\beta)},
$$

then $H(\beta)>0$, a contradiction. Hence, $\beta^{*}>\tilde{\beta}>-0.45$. 
Remark 3.1 Theorem 3.2 improves the lower bound of $\beta^{*}$ from -0.5 in [9] to -0.45 .

\section{Competing interests}

The authors declare that they have no competing interests.

\section{Authors' contributions}

All authors read and approved the final manuscript.

\section{Acknowledgements}

The authors wish to thank the anonymous referees for their valuable comments. This research was supported by the National Natural Science Foundation of China (Grant No. 11171046) and Scientific Research Foundation of the Education Department of Sichuan Province, China.

Received: 12 August 2012 Accepted: 7 September 2012 Published: 25 September 2012

\section{References}

1. Blasius, H: Grenzschichten in Flüssigkeiten mit kleiner Reibung. Z. Angew. Math. Phys. 56, 1-37 (1908)

2. Aly, EH, Elliott, L, Ingham, DB: Mixed convection boundary-layer flow over vertical surface embedded in a porous medium. Eur. J. Mech. B, Fluids 22(6), 529-543 (2003)

3. Weidman, PD: New solutions for laminar boundary layers with cross flow. Z. Angew. Math. Phys. 48(2), 341-356 (1997)

4. Weyl, H: On the differential equations of the simplest boundary layer problem. Ann. Math. 43, 381-407 (1942)

5. Coppel, WA: On a differential equation of boundary layer theory. Philos. Trans. R. Soc. Lond. 253, 101-136 (1960)

6. Hartman, P: Ordinary Differential Equations. Wiley, New York (1964)

7. Belhachmi, Z, Brighi, B, Taous, K: On the concave solutions of the Blasius equations. Acta Math. Univ. Comen. 69(2), 199-212 (2000)

8. Oleinik, OA, Samokhin, VN: Mathematical Models in Boundary Layer Theory. Chapman and Hall/CRC, Boca Raton (1999)

9. Hussaini, MY, Lakin, WD: Existence and nonuniqueness of similarity solutions of boundary-layer problem. Q. J. Mech. Appl. Math. 39(1), 15-24 (1986)

10. Brighi, B, Fruchard, A, Sari, T: On the Blasius problem. Adv. Differ. Equ. 13(5-6), 509-600 (2008)

11. Yang, GC: An upper bound on the critical value $\beta^{*}$ involved in the Blasius problem. J. Inequal. Appl. 2010, Article ID 960365 (2010)

12. Yang, GC, Lan, KQ: The velocity and shear stress functions of the Falkner-Skan equation arising in boundary layer theory. J. Math. Anal. Appl. 328(2), 1297-1308 (2007)

13. Lan, KQ, Yang, GC: Positive solutions of the Falkner-Skan equation arising in the boundary layer theory. Can. Math. Bull. 51(3), 386-398 (2008)

14. Yang, GC: Existence of solutions of laminar boundary layer equations with decelerating external flows. Nonlinear Anal. TMA 72(3-4), 2063-2075 (2010)

15. Yang, GC, Lan, KQ: Nonexistence of the reversed flow solutions of the Falkner-Skan equations. Nonlinear Anal. TMA 74(16), 5327-5339 (2011)

doi:10.1186/1029-242X-2012-208

Cite this article as: Yang et al.: On the shear stress function and the critical value of the Blasius problem. Journal of Inequalities and Applications 2012 2012:208.

\section{Submit your manuscript to a SpringerOpen ${ }^{\circ}$ journal and benefit from:}

- Convenient online submission

Rigorous peer review

- Immediate publication on acceptance

- Open access: articles freely available online

- High visibility within the field

- Retaining the copyright to your article 